\title{
Noncommutative Differential Geometry of Generalized Weyl Algebras
}

\author{
Tomasz BRZEZIŃSKI †
}

$\dagger$ Department of Mathematics, Swansea University, Singleton Park, Swansea SA2 8PP, UK

E-mail: T.Brzezinski@swansea.ac.uk

$\ddagger$ Department of Mathematics, University of Biatystok, K. Ciotkowskiego 1M, 15-245 Biatystok, Poland

Received February 29, 2016, in final form June 14, 2016; Published online June 23, 2016

http://dx.doi.org/10.3842/SIGMA.2016.059

\begin{abstract}
Elements of noncommutative differential geometry of $\mathbb{Z}$-graded generalized Weyl algebras $\mathcal{A}(p ; q)$ over the ring of polynomials in two variables and their zero-degree subalgebras $\mathcal{B}(p ; q)$, which themselves are generalized Weyl algebras over the ring of polynomials in one variable, are discussed. In particular, three classes of skew derivations of $\mathcal{A}(p ; q)$ are constructed, and three-dimensional first-order differential calculi induced by these derivations are described. The associated integrals are computed and it is shown that the dimension of the integral space coincides with the order of the defining polynomial $p(z)$. It is proven that the restriction of these first-order differential calculi to the calculi on $\mathcal{B}(p ; q)$ is isomorphic to the direct sum of degree 2 and degree -2 components of $\mathcal{A}(p ; q)$. A Dirac operator for $\mathcal{B}(p ; q)$ is constructed from a (strong) connection with respect to this differential calculus on the (free) spinor bimodule defined as the direct sum of degree 1 and degree -1 components of $\mathcal{A}(p ; q)$. The real structure of KO-dimension two for this Dirac operator is also described.
\end{abstract}

Key words: generalized Weyl algebra; skew derivation; differential calculus; principal comodule algebra; strongly graded algebra; Dirac operator

2010 Mathematics Subject Classification: 16S38; 58B32; 58B34

\section{Introduction}

In this paper we continue the study initiated in [5] of some aspects of the noncommutative geometry of a class of degree-one generalized Weyl algebras [1] or rank-one hyperbolic algebras [19] over the polynomial ring in one and two variables. We denote these algebras respectively by $\mathcal{B}(p ; q)$ and $\mathcal{A}(p ; q)$, where $q$ is a non-zero element of the ground field $\mathbb{K}$ (of characteristic 0 ) and $p$ is a polynomial in one variable. $\mathcal{B}(p ; q)$ can be interpreted as the coordinate algebra of a noncommutative surface, while, as shown in [5], $\mathcal{A}(p ; q)$ is a noncommutative principal circle bundle over $\mathcal{B}(p ; q)$ provided zero is not a root of $p$. In algebraic terms this means that $\mathcal{A}(p ; q)$ is a strongly $\mathbb{Z}$-graded algebra [13] (or a Clifford system [12]) and $\mathcal{B}(p ; q)$ is isomorphic to the degree-zero part of $\mathcal{A}(p ; q)$. Perhaps best-known examples of such algebras are the standard quantum Podleś sphere [22] with the quantum $\mathrm{SU}_{q}(2)$-group [24] as a bundle over it (in this case $p$ is a linear polynomial) which together form the quantum Hopf fibration [9]. More recent examples include quantum teardrops [7] with quantum lens spaces [15] as bundles over them.

While in [5] we concentrated on establishing that $\mathcal{A}(p ; q)$ are principal comodule algebras [8] or noncommutative principal bundles [9] over $\mathcal{B}(p ; q)$ and on some homological properties of $\mathcal{A}(p ; q)$, the aim of the present article is to describe differential aspects of these algebras. We begin by introducing three families of skew derivations on $\mathcal{A}(p ; q)$. Each family is labeled by a scalar, and they include derivations of $\mathbb{Z}$-degrees -2 (denoted by $\partial_{+}$), 0 (denoted by $\partial_{0}$ ) and 2 (denoted by $\left.\partial_{-}\right)$, respectively. The Leibniz rule for each of these skew derivations is twisted by an 
automorphism, denoted by $\sigma_{+}, \sigma_{0}$ and $\sigma_{-}$, respectively. Choosing one member of each family, we form the system $\left(\partial_{i}, \sigma_{i}\right), i= \pm, 0$ which is a (diagonal) free twisted multi-derivation of size 3 and thus defines a genuine derivation $d$ with values in a free left (and right) $\mathcal{A}(p ; q)$-module $\Omega$; see, e.g., [6]. As a left $\mathcal{A}(p ; q)$-module, $\Omega$ is isomorphic to $\mathcal{A}(p ; q)^{3}$, while the right $\mathcal{A}(p ; q)$-action is twisted by automorphisms $\sigma_{i}$; see (4.1) for the definition of the right action and (4.2) for the definition of $d$. Next we show in Theorem 4.2 that if the algebra $\mathcal{A}(p ; q)$ is regular, i.e., $p(0) \neq 0$ and $p(z)$ is coprime with its $q^{2}$-derivative (see Definition 2.1) and $q^{2} \neq 1$, or $p$ has degree at most one and a non-zero constant term, then $\Omega=\mathcal{A}(p ; q) d(\mathcal{A}(p ; q))$, that is $(\Omega, d)$ is a first-order differential calculus over $\mathcal{A}(p ; q)$. In the complex case, if $q$ and all the coefficients of $p$ are real, $\mathcal{A}(p ; q)$ can be made into a $*$-algebra and $(\Omega, d)$ into a $*$-calculus. In the case of the linear polynomial $p(z)=1-z$ the $*$-calculus $(\Omega, d)$ coincides with the $3 \mathrm{D}$ (left) covariant calculus on $\mathrm{SU}_{q}(2)$ introduced by Woronowicz in [25]. Since the bimodule of one-forms $\Omega$ is free both as a left and right $\mathcal{A}(p ; q)$-module, the cotangent bundle of the noncommutative space described by $\mathcal{A}(p ; q)$ is trivial. Following the procedure described in [6] we associate a divergence or a hom-connection [4] to $(\Omega, d)$, establish that the dimension of its cokernel or the integral space is equal to the degree of $p(z)$ and find a recursive formula that determines the associated integral.

Next we discuss the restriction of $(\Omega, d)$ to the calculus on the degree-zero subalgebra of $\mathcal{A}(p ; q)$ isomorphic to $\mathcal{B}(p ; q)$. We show that in the regular case, the sections of the cotangent bundle are isomorphic to the direct sum of degree -2 and 2 components of $\mathcal{A}(p ; q)$ provided $q^{4} \neq 1$ or $p$ is linear. This interpretation gives a natural splitting of the calculus on $\mathcal{B}(p ; q)$ into holomorphic and anti-holomorphic forms. Furthermore, it allows one to apply techniques developed by Beggs and Majid in [2] to construct Dirac operators on $\mathcal{B}(p ; q)$. The sections of the spinor bundle are isomorphic to the direct sum of degree -1 and 1 components of $\mathcal{A}(p ; q)$. We show that this direct sum is free as a left (or right) $\mathcal{B}(p ; q)$-module, a result that mimics exactly what happens in the case of the standard quantum 2-sphere [20]. Again following the Beggs-Majid procedure from [2] we equip thus constructed Dirac operators with real structures, and determine their KO-dimension to be 2 (modulo 8). This further enforces heuristic understanding of regular generalized Weyl algebras $\mathcal{B}(p ; q)$ as coordinate algebras of noncommutative Riemann surfaces.

Throughout, all algebras are associative, unital and defined over a field $\mathbb{K}$ of characteristic 0 .

\section{Preliminaries}

Definition 2.1. Let $\mathbb{K}$ be a field of characteristic $0, q \in \mathbb{K}$, and let $p(z)$ be a polynomial in one variable with coefficients from $\mathbb{K}$. Let

$$
p_{q}(z):=\frac{p(q z)-p(z)}{(q-1) z}
$$

denote the $q$-derivative of $p$. We say that $p$ is a $q$-separable polynomial if $p(z)$ is coprime with $z p_{q}(z)$.

It is clear from Definition 2.1 that 0 cannot be a root of a $q$-separable polynomial. Furthermore, the properties of greatest common divisors imply that if $q \neq 1$, a non-constant polynomial $p(z)$ is $q$-separable if and only if it has no common factors with $p(q z)$ other than scalars. In case of $q=1$ in which the $q$-derivative (2.1) is the usual formal derivative of polynomials, a 1-separable polynomial is simply a separable polynomial with a non-zero constant term.

In the following definition the algebras studied in this paper are introduced.

Definition 2.2. Let $\mathbb{K}$ be a field, $q \neq 0$ an element of $\mathbb{K}$, and let $p$ be a polynomial in one variable with coefficients from $\mathbb{K}$. 
(1) The algebra $\mathcal{A}(p ; q)$ is an affine algebra generated by $x_{+}, x_{-}, z_{-}, z_{+}$subject to relations:

$$
\begin{aligned}
& z_{+} z_{-}=z_{-} z_{+}, \quad x_{+} z_{ \pm}=q^{-1} z_{ \pm} x_{+}, \quad x_{-} z_{ \pm}=q z_{ \pm} x_{-}, \\
& x_{+} x_{-}=p\left(z_{+} z_{-}\right), \quad x_{-} x_{+}=p\left(q^{2} z_{-} z_{+}\right) .
\end{aligned}
$$

(2) The algebra $\mathcal{B}(p ; q)$ is an affine algebra generated by $x, y, z$ subject to relations:

$$
x z=q^{2} z x, \quad y z=q^{-2} z y, \quad x y=q^{2} z p\left(q^{2} z\right), \quad y x=z p(z) .
$$

(3) We say that $\mathcal{A}(p ; q)$ (resp. $\mathcal{B}(p ; q))$ is regular provided $p$ is a $q^{2}$-separable polynomial.

Both $\mathcal{A}(p ; q)$ and $\mathcal{B}(p ; q)$ are degree-one generalized Weyl algebras $[1] ; \mathcal{A}(p ; q)$ is defined over the ring $\mathbb{K}\left[z_{+}, z_{-}\right]$, while $\mathcal{B}(p ; q)$ extends $\mathbb{K}[z]$.

The algebra $\mathcal{A}(p ; q)$ is a $\mathbb{Z}$-graded algebra with the grading given on the generators by $\left|z_{ \pm}\right|=$ $\left|x_{ \pm}\right|= \pm 1$. The degree-zero subalgebra $\mathcal{A}(p ; q)_{0}$ is isomorphic to $\mathcal{B}(p ; q)$ by the map

$$
\Theta: \mathcal{B}(p ; q) \rightarrow \mathcal{A}(p ; q)_{0}, \quad x \mapsto x_{-} z_{+}, \quad y \mapsto z_{-} x_{+}, \quad z \mapsto z_{-} z_{+}
$$

If $\mathbb{K}=\mathbb{C}, q$ is a real number and $p$ has real coefficients, then both $\mathcal{A}(p ; q)$ and $\mathcal{B}(p ; q)$ can be made into $*$-algebras by setting

$$
z_{-}^{*}=z_{+}, \quad x_{-}^{*}=x_{+} ; \quad z^{*}=z, \quad x^{*}=y .
$$

The map $\Theta$ is then an isomorphism of $*$-algebras. In the case of the ( $q^{2}$-separable $)$ polynomial

$$
p(z)=\prod_{k=0}^{l-1}\left(1-q^{-2 \frac{k}{l}} z\right),
$$

the $*$-algebra $\mathcal{A}(p ; q)$ is the coordinate algebra of a quantum lens space [15], while $\mathcal{B}(p ; q)$ is the coordinate algebra of the quantum teardrop [7]. Furthermore, if $l=1, \mathcal{A}(p ; q)$ is the coordinate algebra of the quantum $\mathrm{SU}_{q}(2)$ group [24], while $\mathcal{B}(p ; q)$ is the standard Podleś sphere algebra [22]. Finally, if $p$ is a non-zero constant polynomial, then $x_{+}$is invertible with the inverse proportional to $x_{-}$, hence $\mathcal{A}(p ; q)$ is a $q$-skew Laurent polynomial algebra over the ring of polynomials in two variables. In this case $\mathcal{B}(p ; q)$ is the quantum plane (the quantum polynomial ring in two variables).

As explained in [5, Theorem 3.7], if $p(0) \neq 0$, then $\mathcal{A}(p ; q)$ is a strongly graded algebra, i.e., every homogeneous element of $\mathcal{A}(p ; q)$ of degree $k+l$ can be written as a linear combination of products of elements of degrees $k$ and $l[13,21]$. Equivalently, there exists a mapping (called a strong connection [14])

$$
\ell: \mathbb{Z} \rightarrow \mathcal{A}(p ; q) \otimes \mathcal{A}(p ; q),
$$

such that, for all $n \in \mathbb{Z}, \ell(n) \in \mathcal{A}(p ; q)_{-n} \otimes \mathcal{A}(p ; q)_{n}$ and the composition of $\ell$ with the multiplication in $\mathcal{A}(p ; q)$ is the constant function $n \mapsto 1 \in \mathcal{A}(p ; q)$. In consequence, all the homogeneous components $\mathcal{A}(p ; q)_{n}$ are projective (in fact invertible) modules over the degree-zero subalgebra identified with $\mathcal{B}(p ; q)$. These modules are mutually non-isomorphic, provided $q$ is not a root of unity and $p$ has at least one non-zero root. Geometrically all this means that $\mathcal{A}(p ; q)$ is a noncommutative principal circle bundle over $\mathcal{B}(p ; q)$ and that $\mathcal{A}(p ; q)_{n}$ are (modules of sections of) non-trivial and mutually non-equivalent line bundles over $\mathcal{B}(p ; q)$ [9]. 


\section{Skew derivations on $\mathcal{A}(p ; q)$}

Let $\mathcal{A}$ be an algebra and $\sigma$ an algebra automorphism of $\mathcal{A}$. We say that a linear map $\partial: \mathcal{A} \rightarrow \mathcal{A}$ is a (right) skew $\sigma$-derivation or that $(\partial, \sigma)$ is a (right) skew derivation if, for all $a, b \in \mathcal{A}$,

$$
\partial(a b)=\partial(a) \sigma(b)+a \partial(b) .
$$

Theorem 3.1. Let $\mathcal{A}(p ; q)$ be a generalized Weyl algebra as in Definition 2.2 , and let $z:=z_{+} z_{-}$. Consider linear maps $\sigma_{ \pm}, \sigma_{0}$ of $\mathcal{A}(p ; q)$ defined on the homogeneous elements a $\in \mathcal{A}(p ; q)$ by

$$
\sigma_{ \pm}(a)=q^{|a|} a, \quad \sigma_{0}(a)=q^{2|a|} a,
$$

and the polynomial

$$
c(z):=q p_{q^{2}}(z)=q \frac{p\left(q^{2} z\right)-p(z)}{\left(q^{2}-1\right) z} .
$$

Then:

(1) The maps $\sigma_{ \pm}, \sigma_{0}$ are algebra automorphisms of $\mathcal{A}(p ; q)$.

(2) For all $\alpha_{0} \in \mathbb{K}$, the map $\partial_{0}$ defined on the generators of $\mathcal{A}(p ; q)$ by

$$
\begin{aligned}
& \partial_{0}\left(x_{+}\right)=\alpha_{0} x_{+}, \quad \partial_{0}\left(x_{-}\right)=-q^{-2} \alpha_{0} x_{-} \quad \partial_{0}\left(z_{+}\right)=\alpha_{0} z_{+}, \\
& \partial_{0}\left(z_{-}\right)=-q^{-2} \alpha_{0} z_{-},
\end{aligned}
$$

extends to the whole of $\mathcal{A}(p ; q)$ as a skew $\sigma_{0}$-derivation.

(3) For all $\alpha_{-} \in \mathbb{K}$, the map $\partial_{-}$defined on the generators of $\mathcal{A}(p ; q)$ by

$$
\partial_{-}\left(x_{+}\right)=\partial_{-}\left(z_{+}\right)=0, \quad \partial_{-}\left(x_{-}\right)=\alpha_{-} c(z) z_{+}, \quad \partial_{-}\left(z_{-}\right)=\alpha_{-} x_{+},
$$

extends to the whole of $\mathcal{A}(p ; q)$ as a skew $\sigma_{-}$-derivation.

(4) For all $\alpha_{+} \in \mathbb{K}$, the map $\partial_{+}$defined on the generators of $\mathcal{A}(p ; q)$ by

$$
\partial_{+}\left(x_{-}\right)=\partial_{+}\left(z_{-}\right)=0, \quad \partial_{+}\left(x_{+}\right)=\alpha_{+} c(z) z_{-}, \quad \partial_{+}\left(z_{+}\right)=\alpha_{+} x_{-},
$$

extends to the whole of $\mathcal{A}(p ; q)$ as a skew $\sigma_{+}$-derivation.

(5) The maps $\partial_{0}, \partial_{ \pm}$are $q$-skew derivations, i.e.,

$$
\sigma_{0}^{-1} \circ \partial_{0} \circ \sigma_{0}=\partial_{0}, \quad \sigma_{ \pm}^{-1} \circ \partial_{ \pm} \circ \sigma_{ \pm}=q^{ \pm 2} \partial_{ \pm} .
$$

(6) The maps $\delta_{ \pm}:=\partial_{ \pm} \circ \Theta: \mathcal{B}(p ; q) \rightarrow \mathcal{A}(p ; q)_{\mp 2}$, where $\Theta$ is the isomorphism (2.3), are $\mathcal{A}(p ; q)_{\mp 2}$-valued derivations, while $\partial_{0} \circ \Theta=0$.

Proof. (1) The statement follows immediately from the fact that $\mathcal{A}(p ; q)$ is a graded algebra.

(2) We need to check that the skew derivation property of $\partial_{0}$ is compatible with relations (2.2).

We compute

$$
\begin{aligned}
\partial_{0}\left(z_{-} z_{+}\right) & =\partial_{0}\left(z_{-}\right) \sigma_{0}\left(z_{+}\right)+z_{-} \partial_{0}\left(z_{+}\right)=\alpha_{0}\left(-q^{-2} \times q^{2}+1\right) z_{-} z_{+}=0 \\
& =\alpha_{0}\left(q^{-2}-q^{-2}\right) z_{+} z_{-}=\partial_{0}\left(z_{+}\right) \sigma_{0}\left(z_{-}\right)+z_{+} \partial_{0}\left(z_{-}\right)=\partial_{0}\left(z_{+} z_{-}\right) .
\end{aligned}
$$

This calculation implies further that, for all polynomials $f, \partial_{0}(f(z))=0$. Hence

$$
\partial_{0}\left(x_{+} x_{-}\right)=\partial_{0}\left(x_{+}\right) \sigma_{0}\left(x_{-}\right)+x_{+} \partial_{0}\left(x_{-}\right)=\alpha_{0}\left(q^{-2}-q^{-2}\right) x_{+} x_{-}=0=\partial_{0}(p(z))
$$


and

$$
\partial_{0}\left(x_{-} x_{+}\right)=\partial_{0}\left(x_{-}\right) \sigma_{0}\left(x_{+}\right)+x_{-} \partial_{0}\left(x_{+}\right)=\alpha_{0}\left(-q^{-2} \times q^{2}+1\right) x_{-} x_{+}=0=\partial_{0}\left(p\left(q^{2} z\right)\right) .
$$

In a similar way one easily finds that

$$
\partial_{0}\left(x_{-} z_{+}\right)=0=\partial_{0}\left(q z_{+} x_{-}\right), \quad \partial_{0}\left(z_{-} x_{+}\right)=0=\partial_{0}\left(q x_{+} z_{-}\right) .
$$

Finally,

$$
\partial_{0}\left(x_{+} z_{+}\right)=\partial_{0}\left(x_{+}\right) \sigma_{0}\left(z_{+}\right)+x_{+} \partial_{0}\left(z_{+}\right)=\alpha_{0}\left(q^{2}+1\right) x_{+} z_{+}
$$

and

$$
\begin{aligned}
\partial_{0}\left(z_{+} x_{+}\right) & =\partial_{0}\left(z_{+}\right) \sigma_{0}\left(x_{+}\right)+z_{+} \partial_{0}\left(x_{+}\right)=\alpha_{0}\left(q^{2}+1\right) z_{+} x_{+} \\
& =q \alpha_{0}\left(q^{2}+1\right) x_{+} z_{+}=\partial_{0}\left(q x_{+} z_{+}\right) .
\end{aligned}
$$

In a similar way the compatibility of the $\sigma_{0}$-skew derivation property of $\partial_{0}$ with the last of commutation relations in $\mathcal{A}(p ; q)$, i.e., that $\partial_{0}\left(x_{-} z_{-}\right)=\partial_{0}\left(q z_{-} x_{-}\right)$is checked.

(3) As in (2) we need to check that the skew derivation property of $\partial_{-}$is compatible with relations $(2.2)$. We start with

$$
\begin{aligned}
\partial_{-}\left(x_{-} z_{-}\right) & =\partial_{-}\left(x_{-}\right) \sigma_{-}\left(z_{-}\right)+x_{-} \partial_{-}\left(z_{-}\right)=\alpha_{-}\left(q^{-1} c(z) z_{+} z_{-}+x_{-} x_{+}\right) \\
& =\alpha_{-}\left(\frac{p\left(q^{2} z\right)-p(z)}{q^{2}-1}+p\left(q^{2} z\right)\right)=\alpha_{-} \frac{q^{2} p\left(q^{2} z\right)-p(z)}{q^{2}-1} .
\end{aligned}
$$

On the other hand

$$
\begin{aligned}
\partial_{-}\left(q z_{-} x_{-}\right) & =q \partial_{-}\left(z_{-}\right) \sigma_{-}\left(x_{-}\right)+q z_{-} \partial_{-}\left(x_{-}\right)=\alpha_{-}\left(x_{+} x_{-}+q z_{-} c(z) z_{+}\right) \\
& =\alpha_{-}\left(p(z)+q^{2} \frac{p\left(q^{2} z\right)-p(z)}{q^{2}-1}\right)=\alpha_{-} \frac{q^{2} p\left(q^{2} z\right)-p(z)}{q^{2}-1}=\partial_{-}\left(x_{-} z_{-}\right),
\end{aligned}
$$

as required. Clearly,

$$
\partial_{-}\left(q x_{+} z_{+}\right)=0=\partial_{-}\left(z_{+} x_{+}\right) .
$$

Next

$$
\begin{aligned}
\partial_{-}\left(z_{-} z_{+}\right) & =\partial_{-}\left(z_{-}\right) \sigma_{-}\left(z_{+}\right)+z_{-} \partial_{-}\left(z_{+}\right)=q \alpha_{-} x_{+} z_{+}=\alpha_{-} z_{+} x_{+} \\
& =\partial_{-}\left(z_{+}\right) \sigma_{-}\left(z_{-}\right)+z_{+} \partial_{-}\left(z_{-}\right)=\partial_{-}\left(z_{+} z_{-}\right) .
\end{aligned}
$$

In a similar way one finds

$$
\partial_{-}\left(x_{-} z_{+}\right)=q \alpha_{-} c(z) z_{+}^{2}=\partial_{-}\left(q z_{+} x_{-}\right) \quad \text { and } \quad \partial_{-}\left(z_{-} x_{+}\right)=q \alpha_{-} x_{+}^{2}=\partial_{-}\left(q x_{+} z_{-}\right) .
$$

Finally, observe that, for all polynomials $f$,

$$
\partial_{-}\left(f\left(z_{-}\right)\right)=\alpha_{-} \frac{f\left(q^{-2} z_{-}\right)-f\left(z_{-}\right)}{\left(q^{-2}-1\right) z_{-}} x_{+},
$$

and, since $\partial_{-}\left(z_{+}\right)=0$,

$$
\partial_{-}(f(z))=\alpha_{-} \frac{f\left(q^{-2} z\right)-f(z)}{\left(q^{-2}-1\right) z} z_{+} x_{+} .
$$


Thus, in particular,

$$
\partial_{-}(p(z))=\alpha_{-} q^{-1} c\left(q^{-2} z\right) z_{+} x_{+} \quad \text { and } \quad \partial_{-}\left(p\left(q^{2} z\right)\right)=\alpha_{-} q c(z) z_{+} x_{+} .
$$

Using these observations one easily finds that

$$
\partial_{-}(p(z))=\partial_{-}\left(x_{+} x_{-}\right) \quad \text { and } \quad \partial_{-}\left(p\left(q^{2} z\right)\right)=\partial_{-}\left(x_{-} x_{+}\right)
$$

as required.

(4) This statement is proven in a way analogous to statement (3). Alternatively, one can observe that the assignment $x_{ \pm} \mapsto x_{\mp}, z_{ \pm} \mapsto z_{\mp}$, defines an anti-algebra automorphism $\varphi$ of $\mathcal{A}(p ; q)$, under which $\partial_{+} \propto \varphi^{-1} \circ \partial_{-} \circ \varphi$; hence (3) implies (4).

(5) One can easily check that $\partial_{0}$ is a degree-zero map, while $\partial_{ \pm}$are degree $\mp 2$ maps, i.e., for all homogeneous $a$,

$$
\left|\partial_{0}(a)\right|=|a|, \quad\left|\partial_{ \pm}(a)\right|=|a| \mp 2 .
$$

In view of the form of the automorphisms (3.1), equations (3.3) follow immediately.

(6) Since the automorphisms $\sigma_{ \pm}$restricted to the degree-zero subalgebra of $\mathcal{A}(p ; q)$ are the identity maps, the restrictions of skew derivations $\partial_{ \pm}$to this subalgebra satisfy the usual Leibniz rule, and thus so do the $\delta_{ \pm}$by the algebra map property of $\Theta$. Furthermore, since $\partial_{ \pm}$are degree $\mp 2$ maps, the codomains of $\delta_{ \pm}$come out as stated. The calculations of part (2) together with the (skew) derivation property show that $\partial_{0}$ restricted to the degree-zero subalgebra of $\mathcal{A}(p ; q)$ is the zero map, and so is $\delta_{0}$.

\section{Differential and integral calculi on $\mathcal{A}(p ; q)$}

In this section, to the system of skew derivations on $\mathcal{A}(p ; q)$ constructed in Theorem 3.1 we associate an $\mathcal{A}(p ; q)$-bimodule $\Omega$ with a derivation $d: \mathcal{A}(p ; q) \rightarrow \Omega$, and show that $(\Omega, d)$ forms a first-order differential calculus for $\mathcal{A}(p ; q)$. We then proceed to discuss the canonical divergence or hom-connection associated to $(\Omega, d)$, and calculate the corresponding integral and integral space. We assume that the constants $\alpha_{ \pm}, \alpha_{0}$ in Theorem 3.1 are not equal to zero.

Definition 4.1. Let $\mathcal{A}$ be an algebra. By a first-order differential calculus on $\mathcal{A}$ we mean an $\mathcal{A}$-bimodule $\Omega$ together with a $\mathbb{K}$-linear map $d: \mathcal{A} \rightarrow \Omega$ such that

(a) $d$ is an $\Omega$-valued derivation of $\mathcal{A}$, i.e., it satisfies the Leibniz rule: for all $a, b \in \mathcal{A}$,

$$
d(a b)=d(a) b+a d(b)
$$

(b) $\Omega$ satisfies the density condition $\Omega=\mathcal{A} d(\mathcal{A})$, i.e., for all $\omega \in \Omega$, there exists a finite number of elements $a_{i}, b_{i} \in \mathcal{A}$ such that

$$
\omega=\sum_{i} a_{i} d\left(b_{i}\right)
$$

If $\mathcal{A}$ is a complex $*$-algebra, then the calculus $(\Omega, d)$ is said to be a $*$-calculus provided $\Omega$ is equipped with an anti-linear operation $*$ such that, for all $a, b \in \mathcal{A}, \omega \in \Omega$,

$$
(a \omega b)^{*}=b^{*} \omega^{*} a^{*} \quad \text { and } \quad d\left(a^{*}\right)=d(a)^{*} .
$$


Note that due to the Leibniz rule, the density condition can be equivalently stated as $\Omega=$ $d(\mathcal{A}) \mathcal{A}$ or $\Omega=d(\mathcal{A}) \mathcal{A}+\mathcal{A d}(\mathcal{A})$.

Following the construction of [6], fix a finite indexing set $I$, and let $\left(\partial_{i}, \sigma_{i}\right), i \in I$, be a collection of (right) skew derivations on an algebra $\mathcal{A}$. Let $\Omega$ be a free left $\mathcal{A}$-module with a free basis $\omega_{i}, i \in I$. Define the (free) right $\mathcal{A}$-module structure on $\Omega$ by setting

$$
\omega_{i} a:=\sigma_{i}(a) \omega_{i} .
$$

Then the map

$$
d: \mathcal{A} \rightarrow \Omega, \quad a \mapsto \sum_{i \in I} \partial_{i}(a) \omega_{i}
$$

is an $\Omega$-valued derivation of $\mathcal{A}$, i.e., it satisfies condition (a) in Definition 4.1. There is no guarantee in general that the density condition of Definition 4.1 be satisfied, though.

Theorem 4.2. Let $\partial_{0}, \partial_{ \pm}$be skew derivations of a regular generalized Weyl algebra $\mathcal{A}(p ; q)$ defined in Theorem 3.1, with $\alpha_{0}, \alpha_{ \pm} \neq 0$. Set $I=\{-, 0,+\}$, let $\Omega$ be a free left $\mathcal{A}(p ; q)$-module with a basis $\omega_{-}, \omega_{0}, \omega_{+}$, and equip it with the right module structure (4.1). If $q^{2} \neq 1$ or $\operatorname{deg}(p(z)) \leq 1$, then $\Omega$ together with the map $d: \mathcal{A}(p ; q) \rightarrow \Omega$ defined by (4.2) is a first-order calculus on $\mathcal{A}(p ; q)$.

Proof. We start by listing explicitly relations that define $\Omega$ and $d$ on generators. The bimodule structure of $\Omega$ is determined from the relations:

$$
\begin{array}{ll}
\omega_{-} z_{ \pm}=q^{ \pm 1} z_{ \pm} \omega_{-}, & \omega_{-} x_{ \pm}=q^{ \pm 1} x_{ \pm} \omega_{-}, \\
\omega_{0} z_{ \pm}=q^{ \pm 2} z_{ \pm} \omega_{0}, & \omega_{0} x_{ \pm}=q^{ \pm 2} x_{ \pm} \omega_{0}, \\
\omega_{+} z_{ \pm}=q^{ \pm 1} z_{ \pm} \omega_{+}, & \omega_{+} x_{ \pm}=q^{ \pm 1} x_{ \pm} \omega_{+} .
\end{array}
$$

The action of $d$ on the generators of $\mathcal{A}(p ; q)$ comes out as

$$
\begin{aligned}
& d\left(x_{+}\right)=\alpha_{0} x_{+} \omega_{0}+\alpha_{+} c(z) z_{-} \omega_{+}, \\
& d\left(x_{-}\right)=-q^{-2} \alpha_{0} x_{-} \omega_{0}+\alpha_{-} c(z) z_{+} \omega_{-}, \\
& d\left(z_{+}\right)=\alpha_{0} z_{+} \omega_{0}+\alpha_{+} x_{-} \omega_{+}, \\
& d\left(z_{-}\right)=-q^{-2} \alpha_{0} z_{-} \omega_{0}+\alpha_{-} x_{+} \omega_{-},
\end{aligned}
$$

where, as before, we write $z=z_{-} z_{+}$and $c(z)$ is given by (3.2). We need to prove that

$$
\omega_{ \pm}, \omega_{0} \in \mathcal{A}(p ; q) d(\mathcal{A}(p ; q))=\mathcal{A}(p ; q) d(\mathcal{A}(p ; q))+d(\mathcal{A}(p ; q)) \mathcal{A}(p ; q) .
$$

First assume that $q^{2} \neq 1$. Using relations (4.3) and (4.4) one finds that

$$
z_{-} d\left(z_{+}\right)-d\left(z_{+}\right) z_{-}=\left(1-q^{-2}\right) \alpha_{0} z \omega_{0},
$$

and since $\alpha_{0} \neq 0$ and $q^{2} \neq 1$, we conclude that $z \omega_{0} \in \mathcal{A}(p ; q) d(\mathcal{A}(p ; q))$. Again using relations (4.3) and (4.4) one finds that

$$
d\left(x_{+}\right) x_{-}-q^{-1} c(z) z_{-} d\left(z_{+}\right)+q^{2} x_{+} d\left(x_{-}\right)-q z_{+} d\left(z_{-}\right) c(z)=\left(q^{-2}-1\right) \alpha_{0} p(z) \omega_{0},
$$

hence also $p(z) \omega_{0} \in \mathcal{A}(p ; q) d(\mathcal{A}(p ; q))$. Since the polynomial $p(z)$ is $q^{2}$-separable it does not have a factor $z$, and thus, by the Bézout identity there exist polynomials $f(z), g(z)$ such that

$$
f(z) z+g(z) p(z)=1 \text {. }
$$


In the light of this, multiplying from the left $z \omega_{0}$ by $f(z)$ and $p(z) \omega_{0}$ by $g(z)$ we obtain that $\omega_{0} \in \mathcal{A}(p ; q) d(\mathcal{A}(p ; q))$, as required.

If $p(z)=\lambda z+\mu$, with scalar coefficients $\lambda$ and $\mu \neq 0$ (which is necessary for $p$ to be $q^{2}$-regular), then in view of (4.4), (4.3) and (2.2)

$$
x_{-} d\left(x_{+}\right)-q^{2} \lambda z_{-} d\left(z_{+}\right)=\alpha_{0} \mu \omega_{0},
$$

and so $\omega_{0} \in \mathcal{A}(p ; q) d(\mathcal{A}(p ; q))$ with no assumptions on $q$.

In view of equations (4.4), also

$$
c(z) z_{ \pm} \omega_{ \pm}, x_{ \pm} \omega_{ \pm} \in \mathcal{A}(p ; q) d(\mathcal{A}(p ; q))
$$

Multiplying the first pair by $z_{\mp}$ and the second by $x_{\mp}$, and using relations $(2.2)$ we conclude that

$$
z c(z) \omega_{ \pm}, p(z) \omega_{ \pm} \in \mathcal{A}(p ; q) d(\mathcal{A}(p ; q)) .
$$

Since the polynomial $p(z)$ is $q^{2}$-separable, there is a polynomial combination of $p(z)$ and $z c(z)$ that gives 1 , and hence $\omega_{ \pm} \in \mathcal{A}(p ; q) d(\mathcal{A}(p ; q))$. This completes the proof of the density condition and thus also of the theorem.

Remark 4.3. If $\mathcal{A}(p ; q)$ is a complex $*$-algebra as in (2.4), then the calculus described in Theorem 4.2 can be made into a $*$-calculus with the $*$-operation defined on the generators by

$$
\omega_{ \pm}^{*}=\omega_{\mp}, \quad \omega_{0}^{*}=-\omega_{0},
$$

provided $\alpha_{0} \in \mathbb{R}$ and $\alpha_{-}^{*}=q \alpha_{+}$.

Remark 4.4. Up to rescaling of generators or up to making suitable choices for the parameters $\alpha_{0}, \alpha_{ \pm}$, in the case of $p(z)=1-z$, the calculus in Theorem 4.2 coincides with the 3D calculus of Woronowicz on the quantum $\mathrm{SU}_{q}(2)$-group [25].

Remark 4.5. If $\operatorname{deg}(p(z))>1$, the arguments used in the proof of Theorem 4.2 make significant use of the fact that $q^{2} \neq 1$, hence they do not apply to the classical case $q=1$. However, if $p(z)$ is a non-zero constant or linear polynomial with a non-zero constant term, such as for example in the preceding remark, the assertion of Theorem 4.2 remains true if $q=1$. The resulting commutative algebra is the coordinate algebra of a parallelisable manifold (the product of the circle with the plane or the three-sphere), hence the module of sections of the cotangent bundle is free. If $p$ has higher degree, there is no reason why the corresponding manifold should be a parallelisable three-manifold, and thus no reason to expect its cotangent bundle to be (globally) trivial. In short, one can thus say that the $q$-deformation trivializes the cotangent bundle in this case or that noncommutativity forces triviality.

Note also that the if $q \neq \pm 1$, the assertions of Theorem 4.2 hold also for polynomials with repeated roots (as long as they are $q^{2}$-separable) as the $q^{2}$-separability of $p(z)$ does not imply that $p(z)$ has no repeated roots (this implication only holds in the case $q^{2}=1$ ). If $p(z)$ has repeated roots then both in the commutative and noncommutative cases, the algebra $\mathcal{A}(p ; q)$ has infinite global dimension [1], and hence it is not homologically smooth in the sense of [23], i.e., it does not admit a finite-length resolution by finitely generated and projective bimodules. Yet, it admits a globally defined first-order differential calculus. In the light of arguments for the interpretation of a homologically smooth algebra as a coordinate algebra on a smooth noncommutative space [17] the author finds this quite surprising.

The notions recalled in the following definition were introduced in [4] and [6]. 
Definition 4.6. Let $\mathcal{A}$ be an algebra with a first-order differential calculus $(\Omega, d)$. Let $\mathfrak{I}$ denote the space of right $\mathcal{A}$-linear maps $\Omega \rightarrow \mathcal{A}$ equipped with the left and right $\mathcal{A}$-actions:

$$
a \cdot f \cdot b(\omega):=a f(b \omega), \quad \text { for all } \quad a, b \in \mathcal{A}, \quad \omega \in \Omega, \quad f \in \mathfrak{I} .
$$

A divergence or an $\mathcal{A}$-valued hom-connection on $\mathcal{A}$ (relative to the first-order calculus $(\Omega, d)$ ) is a linear map div: $\mathfrak{I} \rightarrow \mathcal{A}$, such that, for all $a \in \mathcal{A}$ and $\xi \in \mathfrak{I}$,

$$
\operatorname{div}(\xi \cdot a)=\operatorname{div}(\xi) a+\xi(d(a))
$$

The cokernel coker(div) is called the integral space associated to div and the cokernel map $\Lambda: \mathcal{A} \rightarrow \operatorname{coker}(\operatorname{div})$ is called the integral on $\mathcal{A}$ associated to div. The triple (I, div, $\Lambda$ ) is called a first-order integral calculus on $\mathcal{A}$.

Proposition 4.7. Let $(\Omega, d)$ be the first-order differential calculus on a regular generalized Weyl algebra $\mathcal{A}=\mathcal{A}(p ; q)$ with the parameter $q$ that is not a root of unity, as described in Theorem 4.2 . Let $\mathfrak{I}=\operatorname{Hom}_{\mathcal{A}}(\Omega, \mathcal{A})$ and set $n=\operatorname{deg}(p(z))$. Define

$$
\operatorname{div}: \mathfrak{I} \rightarrow \mathcal{A}, \quad \xi \mapsto q^{-2} \partial_{-}\left(\xi\left(\omega_{-}\right)\right)+\partial_{0}\left(\xi\left(\omega_{0}\right)\right)+q^{2} \partial_{+}\left(\xi\left(\omega_{+}\right)\right) .
$$

(1) The map div is the unique divergence such that $\operatorname{div}\left(\xi_{i}\right)=0, i=-, 0,+$, where the $\xi_{i}$ form the basis for $\mathfrak{I}$ dual to the $\omega_{i}$, i.e., $\xi_{i}\left(\omega_{j}\right)=\delta_{i j}, i, j \in\{-, 0,+\}$.

(2) The integral $\Lambda$ vanishes on all elements of $\mathcal{A}(p ; q)$ except for the subalgebra $\mathbb{K}[z]$, where it satisfies, for all $f(z) \in \mathbb{K}[z]$,

$$
\Lambda\left(q^{2} p\left(q^{2} z\right) f\left(q^{2} z\right)\right)=\Lambda(p(z) f(z)) .
$$

(3) The integral space associated to div is $n$-dimensional with a basis $v_{i}:=\Lambda\left(z^{i}\right), i=0,1, \ldots$, $n-1$. Furthermore, for all $k \in \mathbb{N}$,

$$
\Lambda\left(z^{n+k}\right)=\sum_{i=0}^{n-1} \frac{[i+1]}{[n+k+1]} \beta_{i}^{k} v_{i}
$$

where

$$
[l]:=\frac{1-q^{2 l}}{1-q^{2}}=1+q^{2}+\cdots+q^{2 l-2}, \quad l \in \mathbb{N},
$$

denote $q^{2}$-integers, and the coefficients $\beta_{i}^{k}$ are determined from the recurrence relations as follows. Let $\hat{p}(z)$ be the monic polynomial associated to $p(z)$ and write

$$
\hat{p}(z)=z^{n}-\sum_{i=0}^{n-1} \mu_{i} z^{i} .
$$

Then

$$
\beta_{i}^{k}=\sum_{j=1}^{n} \mu_{n-j} \beta_{i}^{k-j}+\mu_{i-k}, \quad \mu_{l}=\beta_{i}^{l}=0 \quad \text { if } \quad l<0 .
$$

Proof. (1) Since, by Theorem 3.1, the calculus $(\Omega, d)$ is associated to $q$-skew derivations $\partial_{i}$, the assertion follows from [6, Theorem 3.4]. 
(2) Using the definition of $\partial_{0}$ and the fact that it is a skew derivation one easily finds that, for all $m>0$,

$$
\partial_{0}\left(x_{+}^{n}\right)=\alpha_{0}[n] x_{+}^{n}, \quad \partial_{0}\left(z_{+}^{n}\right)=\alpha_{0}[n] z_{+}^{n},
$$

where the notation (4.8) is used, and hence

$$
\partial_{0}\left(x_{+}^{n} z_{+}^{m}\right)=\alpha_{0}[m+n] x_{+}^{n} z_{+}^{m} .
$$

In a similar way,

$$
\partial_{0}\left(x_{-}^{n} z_{-}^{m}\right)=\alpha_{0} q^{-2(m+n-1)}[m+n] x_{-}^{n} z_{-}^{m} .
$$

A linear basis for $\mathcal{A}(p ; q)$ consists of $x_{-}^{n} z_{+}^{m} z_{-}^{l}, x_{+}^{n} z_{+}^{m} z_{-}^{l}$. Since the degree-zero subalgebra $\mathcal{B}$ of $\mathcal{A}(p ; q)$ is isomorphic to $\mathcal{B}(p ; q)$, and hence it is generated by $x=x_{-} z_{+}, y=z_{-} x_{+}, z=z_{-} z_{+}$, for all positive $k$, the degree $k$-part of $\mathcal{A}(p ; q)$ is generated by

$$
x_{+}^{k}, x_{+}^{k-1} z_{+}, \ldots, x_{+} z_{+}^{k-1}, z_{+}^{k},
$$

as a $\mathcal{B}$-module. Similarly, for a negative $k$, as a $\mathcal{B}$-module, the degree $k$ component of $\mathcal{A}(p ; q)$ is generated by

$$
x_{-}^{-k}, x_{-}^{-k-1} z_{-}, \ldots, x_{-} z_{-}^{-k-1}, z_{-}^{-k} .
$$

By statement (6) of Theorem 3.1, $\partial_{0}$ restricted to $\mathcal{B}$ is the zero map, and thus, being a skew derivation twisted by an automorphism that is the identity on $\mathcal{B}$, it is a $\mathcal{B}$-bimodule map. Therefore, in view of formulae (4.11), (4.12) and (4.5),

$$
\bigoplus_{k \in \mathbb{Z} \backslash\{0\}} \mathcal{A}(p ; q)_{k} \subseteq \operatorname{Im}\left(\partial_{0}\right) \subseteq \operatorname{Im}(\operatorname{div})=\operatorname{ker} \Lambda .
$$

Next, using the definition of $\partial_{+}$and the fact that it is a skew derivation, one easily finds that, for all $n>0$,

$$
\partial_{+}\left(z_{+}^{n}\right)=\alpha_{+} q^{-n+1}[n] x_{-} z_{+}^{n-1} .
$$

Since $\partial_{+}\left(x_{-}\right)=\partial_{+}\left(z_{-}\right)=0$, we can compute

$$
\begin{aligned}
\partial_{+}\left(x_{-}^{k} z_{+}^{k+l+2} z_{-}^{l}\right) & =q^{-l} x_{-}^{k} \partial_{+}\left(z_{+}^{k+l+2}\right) z_{-}^{l}=\alpha_{+} q^{-k-2 l-1}[k+l+2] x_{-}^{k+1} z_{+}^{k+1} z_{+}^{l} z_{-}^{l} \\
& =\alpha_{+} q^{-2 l+\frac{(k-1) k}{2}-1}[k+l+2] x^{k+1} z^{l} .
\end{aligned}
$$

Therefore, for all $k, l \in \mathbb{N}$,

$$
x^{k+1} z^{l} \in \operatorname{Im}\left(\partial_{+}\right) \subseteq \operatorname{Im}(\operatorname{div})=\operatorname{ker} \Lambda .
$$

Arguing in a similar way but with $\partial_{-}$instead of $\partial_{+}$, one finds that

$$
y^{k+1} z^{l} \in \operatorname{Im}\left(\partial_{-}\right) \subseteq \operatorname{Im}(\operatorname{div})=\operatorname{ker} \Lambda .
$$

Putting (4.13), (4.14) and (4.15) together we can conclude that $\Lambda$ could be non-zero only on polynomials in $z$.

In view of the definitions of the divergence (4.5) and skew derivations $\partial_{ \pm}$, the only way to obtain a polynomial in $z$ in the image of div is to apply $\partial_{-}$to $x_{-} z_{-} f(z)$ or $\partial_{+}$to $x_{+} z_{+} g(z)$, where $f(z), g(z) \in \mathbb{K}[z]$. This gives

$$
\partial_{-}\left(x_{-} z_{-} f(z)\right)=\frac{\alpha_{-}}{q^{2}-1}\left(q^{2} p\left(q^{2} z\right) f\left(q^{2} z\right)-p(z) f(z)\right)
$$


and

$$
\partial_{+}\left(x_{+} z_{+} g(z)\right)=\frac{\alpha_{+}}{q^{2}-1}\left(q^{2} p\left(q^{2} z\right) g(z)-p(z) g\left(q^{-2} z\right)\right) .
$$

Therefore, the only polynomials in $z$ contained in $\operatorname{Im}(\operatorname{div})=\operatorname{ker} \Lambda$ have the form

$$
q^{2} p\left(q^{2} z\right) f\left(q^{2} z\right)-p(z) f(z), \quad f(z) \in \mathbb{K}[z] .
$$

This proves the equation (4.6).

(3) Equation (4.6) gives a recurrence relation for the values of $\Lambda$ on powers of $z$. Clearly, (4.6) remains true if $p$ is replaced by the associated monic $\hat{p}$, and when the latter is written as in (4.9), then the formula (4.6), evaluated at $f(z)=z^{k}, k \in \mathbb{N}$, gives

$$
\Lambda\left(z^{n+k}\right)=\frac{1}{q^{2 n+2 k+2}-1} \sum_{i=0}^{n-1}\left(q^{2 k+2 i+2}-1\right) \mu_{i} \Lambda\left(z^{k+i}\right)=\sum_{i=0}^{n-1} \frac{[i+k+1]}{[n+k+1]} \mu_{i} \Lambda\left(z^{k+i}\right) .
$$

As the recurrence relation (4.16) has order $n$ and $\mu_{0} \neq 0$, its space of solutions, which coincides with the integral space for $\Lambda$, is $n$-dimensional as stated. Its basis can be chosen as

$$
v_{0}=\Lambda(1), v_{1}=\Lambda(z), \ldots, v_{n-1}=\Lambda\left(z^{n-1}\right) .
$$

Using (4.16) repeatedly, and observing that $\Lambda\left(z^{k+i}\right)$ has the $q^{2}$-integer $[i+k+1]$ in the denominator, which cancels out $[i+k+1]$ in (4.16) one realizes that the expression for $\Lambda\left(z^{n+k}\right)$ in terms of the $v_{i}$ depends on $q^{2}$-rational numbers precisely as in (4.7). Then the formula (4.10) for $q$-independent coefficients is obtained and verified inductively.

Example 4.8. Let us consider a regular generalized Weyl algebra $\mathcal{A}(p ; q)$ with $q$ not a root of unity and

$$
p(z)=z^{2}-1 .
$$

Then $\mu_{0}=1$ and $\mu_{1}=0$ and the recurrence relations (4.10) take the form

$$
\beta_{0}^{0}=\beta_{1}^{1}=1, \quad \beta_{1}^{0}=\beta_{0}^{1}=0, \quad \beta_{i}^{k}=\beta_{i}^{k-2}, \quad \text { for all } \quad k>1 .
$$

Thus, by (4.7) the integral associated to the calculus $\Omega$ is

$$
\Lambda\left(z^{k}\right)= \begin{cases}\frac{1}{[k+1]} \Lambda(1), & \text { if } k \text { is even, } \\ \frac{[2]}{[k+1]} \Lambda(z), & \text { if } k \text { is odd },\end{cases}
$$

and zero on all other elements of the basis $x_{-}^{n} z_{+}^{m} z_{-}^{l}, x_{+}^{n} z_{+}^{m} z_{-}^{l}$ for $\mathcal{A}(p ; q)$.

Example 4.9. Let us consider a regular generalized Weyl algebra $\mathcal{A}(p ; q)$ with $q$ not a root of unity and

$$
p(z)=(z-1)^{2} .
$$

Then $\mu_{0}=-1$ and $\mu_{1}=2$ and the recurrence relations (4.10) take the form

$$
\beta_{0}^{0}=-1, \quad \beta_{1}^{0}=2, \quad \beta_{0}^{1}=-2, \quad \beta_{1}^{1}=3, \quad \beta_{i}^{k}=2 \beta_{i}^{k-1}-\beta_{i}^{k-2}, \quad \text { for all } k>1,
$$

which have solutions

$$
\beta_{0}^{k}=-(k+1), \quad \beta_{1}^{k}=k+2 .
$$

Therefore,

$$
\Lambda\left(z^{k}\right)=-\frac{k-1}{[k+1]} \Lambda(1)+\frac{[2]}{[k+1]} k \Lambda(z),
$$

and zero on all other elements of the basis $x_{-}^{n} z_{+}^{m} z_{-}^{l}, x_{+}^{n} z_{+}^{m} z_{-}^{l}$ for $\mathcal{A}(p ; q)$. 


\section{Differential calculus and Dirac operators on $\mathcal{B}(p ; q)$}

In this section we identify the algebra $\mathcal{B}(p ; q)$ with the degree-zero subalgebra of $\mathcal{A}(p ; q)$ via the isomorphism (2.3), and describe a first-order differential calculus and Dirac operators on $\mathcal{B}(p ; q)$. We assume that the parameters $\alpha_{ \pm}$in Theorem 3.1 are non-zero.

Theorem 5.1. Let $\mathcal{A}(p ; q)$ be a regular generalized Weyl algebra, with $q$ not a quartic root of unity or otherwise $\operatorname{deg}(p(z)) \leq 1$, and let $(\Omega, d)$ be the first-order differential calculus defined in Theorem 4.2. View $\mathcal{B}(p ; q)$ as a degree-zero subalgebra of $\mathcal{A}(p ; q)$ and let

$$
\bar{\Omega}:=\mathcal{B}(p ; q) d(\mathcal{B}(p ; q)) \mathcal{B}(p ; q)
$$

be the calculus on $\mathcal{B}(p ; q)$ induced from $(\Omega, d)$. Then

$$
\bar{\Omega} \cong \mathcal{A}(p ; q)_{2} \oplus \mathcal{A}(p ; q)_{-2},
$$

as $\mathcal{B}(p ; q)$-bimodules.

Proof. Since $\omega_{ \pm}$are generators of degrees \pm 2 , commuting with elements of $\mathcal{B}(p ; q)$, and $\mathcal{B}(p ; q)$ $\subseteq \operatorname{ker}\left(\partial_{0}\right)$

$$
\bar{\Omega} \subseteq \mathcal{A}(p ; q)_{2} \omega_{-} \oplus \mathcal{A}(p ; q)_{-2} \omega_{+} .
$$

As a $\mathcal{B}(p ; q)$-module, $\mathcal{A}(p ; q)_{2}$ is generated by $z_{+}^{2}, x_{+}^{2}, z_{+} x_{+}$while $\mathcal{A}(p ; q)_{-2}$ is generated by $z_{-}^{2}, x_{-}^{2}, z_{-} x_{-}$, hence to show that the above inclusion is an equality, it suffices to prove that

$$
z_{+}^{2} \omega_{-}, x_{+}^{2} \omega_{-}, z_{+} x_{+} \omega_{-}, x_{-}^{2} \omega_{+}, z_{-}^{2} \omega_{+}, z_{-} x_{-} \omega_{+} \in \bar{\Omega} .
$$

First we consider the case $q^{4} \neq 1$. Using the definition of $d$ in terms of skew derivations $\partial_{ \pm}$ and $\partial_{0}$ or using equations (4.4) one easily finds that

$$
\begin{aligned}
& d(x)=q \alpha_{-} c(z) z_{+}^{2} \omega_{-}+\alpha_{+} x_{-}^{2} \omega_{+}, \\
& d(y)=q \alpha_{-} x_{+}^{2} \omega_{-}+\alpha_{+} c(z) z_{-}^{2} \omega_{+}, \\
& d(z)=\alpha_{-} z_{+} x_{+} \omega_{-}+\alpha_{+} z_{-} x_{-} \omega_{+} .
\end{aligned}
$$

Therefore, since the generators $\omega_{ \pm}$commute with all elements of $\mathcal{B}(p ; q)$, we conclude that

$$
\begin{aligned}
& z d(x)-d(x) z=\left(1-q^{4}\right) \alpha_{+} z x_{-}^{2} \omega_{+}, \\
& z d(x)=q \alpha_{-} z c(z) z_{+}^{2} \omega_{-}+\alpha_{+} z x_{-}^{2} \omega_{+}, \\
& d(z) x=\alpha_{-} p(z) z_{+}^{2} \omega_{-}+q^{2} \alpha_{+} z x_{-}^{2} \omega_{+} .
\end{aligned}
$$

Equation (5.3a) implies that $z x_{-}^{2} \omega_{+} \in \bar{\Omega}$, hence also $z c(z) z_{+}^{2} \omega_{-}, p(z) z_{+}^{2} \omega_{-} \in \bar{\Omega}$, by (5.3b) and (5.3c). Since $p(z)$ is $q^{2}$-separable, the latter implies that $z_{+}^{2} \omega_{-} \in \bar{\Omega}$ and, in view of (5.2a), $x_{-}^{2} \omega_{+} \in \bar{\Omega}$. In a similar way one finds that

$$
\begin{aligned}
& z d(y)-d(y) z=\left(q-q^{-3}\right) \alpha_{-} z x_{+}^{2} \omega_{-}, \\
& y d(z)=q^{-1} \alpha_{-} z x_{+}^{2} \omega_{-}+q^{-1} \alpha_{+} p(z) z_{-}^{2} \omega_{+}, \\
& z d(y)=q \alpha_{-} z x_{+}^{2} \omega_{-}+\alpha_{+} z c(z) z_{-}^{2} \omega_{+} .
\end{aligned}
$$

By (5.4a), $z x_{+}^{2} \omega_{-} \in \bar{\Omega}$, hence also $z c(z) z_{-}^{2} \omega_{+}, p(z) z_{-}^{2} \omega_{+} \in \bar{\Omega}$, by (5.4b) and (5.4c). Since $p(z)$ is $q^{2}$-separable, the latter implies that $z_{-}^{2} \omega_{+} \in \bar{\Omega}$ and, in view of $(5.2 \mathrm{~b}), x_{+}^{2} \omega_{-} \in \bar{\Omega}$. Finally,

$$
\alpha_{+} p(z) z_{-} x_{-} \omega_{+}=y d(x)-\frac{q^{-1} c\left(q^{-2} z\right)}{1-q^{-4}}\left(z d(z)-q^{-2} d(z) z\right)
$$


and

$$
\alpha_{+} z z_{-} x_{-} \omega_{+}=\frac{1}{1-q^{4}}\left(z d(z)-q^{2} d(z) z\right)
$$

hence $z_{-} x_{-} \omega_{+} \in \bar{\Omega}$, since the polynomial $p(z)$, being $q^{2}$-separable, does not contain the factor $z$. In view of (5.2c), also $z_{+} x_{+} \omega_{-} \in \bar{\Omega}$.

If $p(z)=\lambda z+\mu, \lambda, \mu \in \mathbb{K}, \mu \neq 0$, then equations (5.2) and the fact that generators $\omega_{ \pm}$ commute with all elements of $\mathcal{B}(p ; q)$ yield

$$
\alpha_{-} \mu z_{+}^{2} \omega_{-}=d(z) x-q^{-2} d(x) z, \quad \alpha_{+} \mu z_{-}^{2} \omega_{+}=q y d(z)-q^{-1} z d(y)
$$

and

$$
\alpha_{-} \mu z_{+} x_{+} \omega_{-}=q^{-2} x d(y)-q^{2} \lambda z d(z),
$$

hence also in this case all the required forms are in $\bar{\Omega}$. Since $\omega_{ \pm}$are $(\mathcal{B}(p ; q)$-central) free generators, we obtain the required isomorphism of $\mathcal{B}(p ; q)$-bimodules (5.1).

Theorem 5.1 shows that the $\mathcal{B}(p ; q)$-bimodule $\mathcal{A}(p ; q)_{2} \oplus \mathcal{A}(p ; q)_{-2}$ plays the role of sections of the cotangent bundle over the quantum surface with the coordinate algebra $\mathcal{B}(p ; q)$. The direct sum decomposition on the right hand side of (5.1) can be interpreted as the decomposition of oneforms into holomorphic and anti-holomorphic parts. This is particularly justified in case $\mathcal{A}(p ; q)$ and $\mathcal{B}(p ; q)$ are equipped with the $*$-algebra structure $(2.4)$ and $\Omega$ (and hence also $\bar{\Omega}$ ) is a $*$ calculus as in Remark 4.3. Following this, we write

$$
\bar{\Omega}^{1,0}=\mathcal{A}(p ; q)_{-2} \omega_{+}, \quad \bar{\Omega}^{0,1}=\mathcal{A}(p ; q)_{2} \omega_{-} .
$$

Theorem 5.1 shows further that the module of horizontal forms

$$
\Omega_{\mathrm{hor}}:=\mathcal{A}(p ; q) d(\mathcal{B}(p ; q)) \mathcal{A}(p ; q),
$$

of the principal circle bundle $\mathcal{A}(p ; q)$ over $\mathcal{B}(p ; q)$ is generated by $\omega_{ \pm}$. The one-form $\omega_{0}$ generates vertical forms. We write $\pi$ for the projection of $\Omega$ onto $\Omega_{\text {hor }}$. In particular, for all $a \in \mathcal{A}(p ; q)$,

$$
\pi(d(a))=\partial_{+}(a) \omega_{+}+\partial_{-}(a) \omega_{-} .
$$

We construct a Dirac operator on $\mathcal{B}(p ; q)$ by following the procedure of Beggs and Majid [2], which, in this particular case, is the generalization of a method employed in [20]. To start with, we identify the sections of a spinor bundle with the $\mathcal{B}(p ; q)$-bimodule $\mathcal{A}(p ; q)_{1} \oplus \mathcal{A}(p ; q)_{-1}$. More precisely, we set

$$
\mathcal{S}_{+}=\mathcal{A}(p ; q)_{-1} \mathrm{~s}_{+}, \quad \mathcal{S}_{-}=\mathcal{A}(p ; q)_{1} \mathrm{~s}_{-}, \quad \mathcal{S}=\mathcal{S}_{+} \oplus \mathcal{S}_{-},
$$

where $\mathbf{s}_{ \pm}$are formally understood as (central, i.e., commuting with elements of $\mathcal{B}(p ; q)$ ) generators that distinguish components of the direct sum. In contrast to the cotangent bundle, the spinor bundle is trivial.

Lemma 5.2. The left (resp. right) $\mathcal{B}(p ; q)$-module $\mathcal{A}(p ; q)_{1} \oplus \mathcal{A}(p ; q)_{-1}$ is free.

Proof. Define

$$
\begin{aligned}
& \ell(1):=\frac{1}{p(0)}\left(\frac{p(0)-p\left(q^{2} z\right)}{z} z_{-} \otimes z_{+}+\left(-q x_{-}\right) \otimes\left(-q^{-1} x_{+}\right)\right) \in \mathcal{A}(p ; q)_{-1} \otimes \mathcal{A}(p ; q)_{1}, \\
& \ell(-1):=\frac{1}{p(0)}\left(z_{+} \otimes \frac{p(0)-p(z)}{z} z_{-}+x_{+} \otimes x_{-}\right) \in \mathcal{A}(p ; q)_{1} \otimes \mathcal{A}(p ; q)_{-1} .
\end{aligned}
$$


Relations (2.2) immediately imply that the multiplication applied to both $\ell(1)$ and $\ell(-1)$ gives 1 , hence $\ell(1)$ and $\ell(-1)$ are values of a strong connection in $\mathcal{A}(p ; q)$. Therefore, as explained in [8], an idempotent corresponding to the projective module $\mathcal{A}(p ; q)_{1}$ can be computed as

$$
e(1)=\frac{1}{p(0)}\left(\begin{array}{cc}
p(0)-p\left(q^{2} z\right) & -q z_{+} x_{-} \\
-q^{-1} x_{+} \frac{p(0)-p\left(q^{2} z\right)}{z} z_{-} & x_{+} x_{-}
\end{array}\right)=\frac{1}{p(0)}\left(\begin{array}{cc}
p(0)-p\left(q^{2} z\right) & -x \\
-\frac{p(0)-p(z)}{z} y & p(z)
\end{array}\right),
$$

while an idempotent of $\mathcal{A}(p ; q)_{-1}$, as

$$
e(-1)=\frac{1}{p(0)}\left(\begin{array}{cc}
x_{-} x_{+} & x_{-} z_{+} \\
\frac{p(0)-p(z)}{z} z_{-} x_{+} & p(0)-p(z)
\end{array}\right)=\frac{1}{p(0)}\left(\begin{array}{cc}
p\left(q^{2} z\right) & x \\
\frac{p(0)-p(z)}{z} y & p(0)-p(z)
\end{array}\right) .
$$

Clearly,

$$
e(1)+e(-1)=\left(\begin{array}{ll}
1 & 0 \\
0 & 1
\end{array}\right)
$$

which proves the assertion.

The strong connection forms $\ell(1), \ell(-1)$ listed in the proof of Lemma 5.2 define a connection $\nabla: \mathcal{S} \rightarrow \bar{\Omega} \otimes \mathcal{S}$ on the spinor bundle $\mathcal{S}$ by the formula

$$
\nabla\left(a \mathbf{s}_{+}+b \mathbf{s}_{-}\right)=\pi(d(a)) \ell(-1) \mathbf{s}_{+}+\pi(d(b)) \ell(1) \mathbf{s}_{-},
$$

for all $a$ of degree -1 and $b$ of degree 1. Explicitly,

$$
\begin{aligned}
\nabla\left(a \mathbf{s}_{+}+b \mathbf{s}_{-}\right)= & \frac{q}{p(0)}\left(\partial_{+}(a) z_{+} \omega_{+} \otimes \frac{p(0)-p(z)}{z} z_{-} \mathbf{s}_{+}+\partial_{+}(a) x_{+} \omega_{+} \otimes x_{-} \mathbf{s}_{+}\right) \\
& +\frac{q}{p(0)}\left(\partial_{-}(a) z_{+} \omega_{-} \otimes \frac{p(0)-p(z)}{z} z_{-} \mathbf{s}_{+}+\partial_{-}(a) x_{+} \omega_{-} \otimes x_{-} \mathbf{s}_{+}\right) \\
& +\frac{q^{-1}}{p(0)}\left(\partial_{+}(b) z_{-} \frac{p(0)-p\left(q^{2} z\right)}{z} \omega_{+} \otimes z_{+} \mathbf{s}_{-}+\partial_{+}(b) x_{+} \omega_{+} \otimes x_{+} \mathbf{s}_{-}\right) \\
& +\frac{q^{-1}}{p(0)}\left(\partial_{-}(b) z_{-} \frac{p(0)-p\left(q^{2} z\right)}{z} \omega_{-} \otimes z_{+} \mathbf{s}_{-}+\partial_{-}(b) x_{+} \omega_{-} \otimes x_{+} \mathbf{s}_{-}\right) .
\end{aligned}
$$

The Clifford action $\triangleright$ of $\bar{\Omega}$ on $\mathcal{S}$ is defined, for all $a, b, c_{ \pm} \in \mathcal{A}(p ; q)$ of degrees $|a|=-1,|b|=1$, $\left|c_{ \pm}\right|= \pm 2$, by

$$
\left(c_{-} \omega_{+}+c_{+} \omega_{-}\right) \triangleright\left(a \mathbf{s}_{+}+b \mathbf{s}_{-}\right)=\beta_{+} c_{-} b \mathbf{s}_{+}+\beta_{-} c_{+} a \mathbf{s}_{-},
$$

where $\beta_{+}, \beta_{-}$are (for the time being arbitrary) elements of the field $\mathbb{K}$. A connection together with a Clifford action define the Dirac operator

$$
D:=\triangleright \circ \nabla: \mathcal{S} \rightarrow \mathcal{S}
$$

Using relations (2.2), the Dirac operator corresponding to data (5.6), (5.7), can be computed as

$$
D\left(a \mathbf{s}_{+}+b \mathbf{s}_{-}\right)=\beta_{+} q^{-1} \partial_{+}(b) \mathbf{s}_{+}+\beta_{-} q \partial_{-}(a) \mathbf{s}_{-} .
$$

$D$ is an even Dirac operator with the grading $\mathcal{B}(p ; q)$-bimodule map defined by

$$
\gamma: \mathcal{S} \rightarrow \mathcal{S}, \quad a \mathbf{s}_{+}+b \mathbf{s}_{-} \longmapsto a \mathbf{s}_{+}-b \mathbf{s}_{-},
$$


since

$$
\begin{aligned}
D \circ \gamma\left(a \mathbf{s}_{+}+b \mathbf{s}_{-}\right) & =D\left(a \mathbf{s}_{+}-b \mathbf{s}_{-}\right)=-\beta_{+} q^{-1} \partial_{+}(b) \mathbf{s}_{+}+\beta \partial_{-} q \partial_{-}(a) \mathbf{s}_{-} \\
& =-\gamma\left(\beta_{+} q^{-1} \partial_{+}(b) \mathbf{s}_{+}+\beta_{-} q \partial_{-}(a) \mathbf{s}_{-}\right)=-\gamma \circ D\left(a \mathbf{s}_{+}+b \mathbf{s}_{-}\right),
\end{aligned}
$$

i.e., $D$ anti-commutes with $\gamma$ as required.

Proposition 5.3. Let $\mathbb{K}=\mathbb{C}, q \in(0,1)$ and $p$ be a $q^{2}$-separable polynomial with real coefficients. Equip the complex algebra $\mathcal{A}(p ; q)$ with the *-algebra structure as in (2.4) and let $\Omega$ be the firstorder calculus constructed in Theorem 4.2 with the $*$-structure as in Remark 4.3 . View $\mathcal{B}(p ; q)$ as a $*$-subalgebra of $\mathcal{A}(p ; q)$, let $\mathcal{S}$ be the module of sections of a spinor bundle (5.5), and let $D$ be the Dirac operator (5.8) with the grading $\gamma$ given by (5.9). Choose $\beta_{ \pm}$such that $\beta_{-}^{*} / \beta_{+}<0$, and let $\nu$ be a solution to the equation

$$
\nu^{2}=-q^{3} \frac{\beta_{-}^{*}}{\beta_{+}} .
$$

Then the linear map

$$
J: \mathcal{S} \rightarrow \mathcal{S}, \quad a \mathbf{s}_{+}+b \mathrm{~s}_{-} \longmapsto-\nu^{-1} b^{*} \mathbf{s}_{+}+\nu a^{*} \mathbf{s}_{-},
$$

equips $D$ with a real structure such that $D$ has KO-dimension two.

Proof. In order for $J$ to be a real structure for $D$ (of KO-dimension two) it needs to satisfy the following five conditions:

$$
\begin{aligned}
& J^{2}=-\mathrm{id} \\
& J \circ \gamma=-\gamma \circ J, \\
& J \circ D=D \circ J, \\
& {[u, J v J]=0, \quad \text { for all } \quad u, v \in \mathcal{B}(p ; q),} \\
& {[[D, u], J v J]=0, \quad \text { for all } \quad u, v \in \mathcal{B}(p ; q),}
\end{aligned}
$$

where $[-,-]$ denotes the commutator and elements of $\mathcal{B}(p ; q)$ act on $\mathcal{S}$ by left multiplication; cf. [11]. The first two conditions are easy to check:

$$
J \circ J\left(a \mathbf{s}_{+}+b \mathbf{s}_{-}\right)=J\left(-\nu^{-1} b^{*} \mathbf{s}_{+}+\nu a^{*} \mathbf{s}_{-}\right)=-a \mathbf{s}_{+}-b \mathbf{s}_{-},
$$

by the involution property of $*$, and

$$
\begin{aligned}
& J \circ \gamma\left(a \mathbf{s}_{+}+b \mathbf{s}_{-}\right)=J\left(a \mathbf{s}_{+}-b \mathbf{s}_{-}\right)=\nu^{-1} b^{*} \mathbf{s}_{+}+\nu a^{*} \mathbf{s}_{-}, \\
& \gamma \circ J\left(a \mathbf{s}_{+}+b \mathbf{s}_{-}\right)=\gamma\left(-\nu^{-1} b^{*} \mathbf{s}_{+}+\nu a^{*} \mathbf{s}_{-}\right)=-\nu^{-1} b^{*} \mathbf{s}_{+}-\nu a^{*} \mathbf{s}_{-}=-J \circ \gamma\left(a \mathbf{s}_{+}+b \mathbf{s}_{-}\right),
\end{aligned}
$$

as required.

To check (5.11c) we use the definitions of $D$ and $J$, and compute, for all $a \in \mathcal{A}(p ; q)_{-1}$, $b \in \mathcal{A}(p ; q)_{1}$,

$$
D \circ J\left(a \mathbf{s}_{+}+b \mathbf{s}_{-}\right)=D\left(-\nu^{-1} b^{*} \mathbf{s}_{+}+\nu a^{*} \mathbf{s}_{-}\right)=q^{-1} \beta_{+} \nu \partial_{+}\left(a^{*}\right) \mathbf{s}_{+}-q \beta_{-} \nu^{-1} \partial_{-}\left(b^{*}\right) \mathbf{s}_{-},
$$

and

$$
\begin{aligned}
J \circ D\left(a \mathbf{s}_{+}+b \mathbf{s}_{-}\right) & =J\left(\beta_{+} q^{-1} \partial_{+}(b) \mathbf{s}_{+}+\beta_{-} q \partial_{-}(a) \mathbf{s}_{-}\right) \\
& =-q^{-1} \beta_{-}^{*} \nu^{-1} \partial_{-}(a)^{*} \mathbf{s}_{+}+q \beta_{+}^{*} \nu \partial_{+}(b)^{*} \mathbf{s}_{-} .
\end{aligned}
$$


Note that, in view of the definition (2.4), if $c \in \mathcal{A}(p ; q)$ is a homogeneous element, then $\left|c^{*}\right|=$ $-|c|$. Applying the involution $*$ to the definition of $d$, using the $*$-structure on $\Omega$ in Remark 4.3 and then the definitions of $\sigma_{ \pm}$(3.1) one finds that, for all homogeneous $c \in \mathcal{A}(p ; q)$,

$$
\partial_{+}(c)^{*}=q^{|c|-2} \partial_{-}\left(c^{*}\right), \quad \partial_{-}(c)^{*}=q^{|c|+2} \partial_{+}\left(c^{*}\right) .
$$

Since $|a|=-1$ and $|b|=1$, equality (5.12) can be developed further to give

$$
J \circ D\left(a \mathbf{s}_{+}+b \mathbf{s}_{-}\right)=-q^{2} \nu^{-1} \beta_{-}^{*} \partial_{+}\left(a^{*}\right) \mathbf{s}_{+}+q^{-2} \nu \beta_{+}^{*} \partial_{-}\left(b^{*}\right) \mathbf{s}_{-},
$$

and so $J \circ D=D \circ J$ by (5.10).

To check the order-zero condition (5.11d), take any $a \in \mathcal{A}(p ; q)_{-1}$ and $u, v \in \mathcal{B}(p ; q)$, and compute

$$
\begin{aligned}
{[u, J v J]\left(a \mathbf{s}_{+}\right) } & =u J\left(v J\left(a \mathbf{s}_{+}\right)\right)-J\left(v J\left(u a \mathbf{s}_{+}\right)\right)=\nu\left(u J\left(v a^{*} \mathbf{s}_{-}\right)-J\left(v a^{*} u^{*} \mathbf{s}_{-}\right)\right) \\
& =-u a v^{*} \mathbf{s}_{+}+u a v^{*} \mathbf{s}_{+}=0 .
\end{aligned}
$$

In a similar way one proves that also for all $b \in \mathcal{A}(p ; q)_{1}$ and $u, v \in \mathcal{B}(p ; q)$,

$$
[u, J v J]\left(b \mathbf{s}_{-}\right)=0 .
$$

Finally, as the first step towards proving the order-one condition (5.11e), observe that the skew derivation property of $\partial_{ \pm}$implies that, for all $u \in \mathcal{B}(p ; q), a \in \mathcal{A}(p ; q)_{-1}$ and $b \in \mathcal{A}(p ; q)_{1}$,

$$
[D, u]\left(a \mathbf{s}_{+}+b \mathbf{s}_{-}\right)=\beta_{+} \partial_{+}(u) b \mathbf{s}_{+}+\beta_{-} \partial_{-}(u) a \mathbf{s}_{-},
$$

hence, for all $v \in \mathcal{B}(p ; q)$,

$$
\begin{aligned}
{[[D, u], J v J]\left(a \mathbf{s}_{+}\right) } & =-[D, u]\left(a v^{*} \mathbf{s}_{+}\right)-\beta_{-} J\left(v J\left(\partial_{-}(u) a \mathbf{s}_{-}\right)\right) \\
& =-\beta_{-} \partial_{-}(u) a v^{*} \mathbf{s}_{-}+\beta_{-} \partial_{-}(u) a v^{*} \mathbf{s}_{-}=0,
\end{aligned}
$$

and similarly $[[D, u], J v J]\left(b \mathbf{s}_{-}\right)=0$. The distribution of signs on the right hand sides of relations (5.11a), (5.11b) and (5.11c) indicates that $D$ is a Dirac operator with a real structure of KOdimension two, as stated.

Remark 5.4. There is some level of arbitrariness in the fixing of the KO-dimension of $D$. Choosing the parameters $\beta_{ \pm}$in such a way that $\beta_{-}^{*} / \beta_{+}>0$, will result in the change of the sign on the right hand side of equation (5.11a). This distribution of signs on the right hand sides of relations $(5.11 \mathrm{a}),(5.11 \mathrm{~b})$ and $(5.11 \mathrm{c})$ corresponds to the KO-dimension being 6 (modulo 8).

\section{Outlook}

The paper the reader is presented with herein is concerned with first-order differential (and integral) calculi on a class of generalized Weyl algebras. A detailed study of higher forms on $\mathcal{A}(p ; q)$ and $\mathcal{B}(p ; q)$, both differential and integral, is a natural next step. Every first-order differential calculus admits an extension to a full differential graded algebra. Such a universal extension might be trivial, might lead to the differential structure of classical dimensions or it might be very large, bearing no resemblance to what can be expected from the classical case. In the latter case, further quotients that reduce the dimension of modules of higher forms might be possible. In the cases studied in the present paper, it would be interesting and indeed desired to find out whether the calculus on a regular generalized Weyl algebra $\mathcal{A}(p ; q)$ described in Theorem 4.2 admits a differential graded algebra of 'classical dimensions', i.e., such that the module of two-forms is free of rank 3 and the module of three-forms is free of rank 1 , with 
no higher forms than three-forms (in other words: the calculus admits a volume three-form). Should such a full differential calculus exist, does the divergence constructed in Proposition 4.7 extend to a flat hom-connection so as to produce a complex of integral forms? Finally, is this complex isomorphic to the de Rham complex of differential forms, i.e., is the differential calculus integrable? Since $\mathcal{A}(p ; q)$ is an affine algebra of Gelfand-Kirillov dimension three, the existence of such an isomorphism would establish differential smoothness of $\mathcal{A}(p ; q)$ in the sense of [10].

Similar questions can and should be asked about the first-order differential calculus $(\bar{\Omega}, d)$ on $\mathcal{B}(p ; q)$. In this case, the extension should contain only two-forms, and the module of twoforms should be free of rank one. This is dictated by the Gelfand-Kirillov dimension of $\mathcal{B}(p ; q)$. It is natural to enquire in this case whether the separation of one-forms into holomorphic and anti-holomorphic components carries on to the higher calculus so as to deliver a complex structure on $\mathcal{B}(p ; q)[3,16]$. Again the question about differential smoothness of $\mathcal{B}(p ; q)$ should be addressed.

It might be that in addition to the $q^{2}$-separability another condition on $p$ should be imposed to guarantee the integrability of differential calculi on $\mathcal{A}(p ; q)$ and $\mathcal{B}(p ; q)$, and to establish differential smoothness of these algebras. As explained in [18], generalized Weyl algebras $\mathcal{B}(p ; q)$ are homologically smooth (i.e., they have finite length resolutions by finitely generated projective bimodules) provided the defining polynomial $p$ has no repeated roots (in case of an algebraically closed field this is equivalent to the separability of $p$ ). Should no additional (to $q^{2}$-separability) conditions on $p$ be required or should required additional conditions be different from separability, the regular generalized Weyl algebras $\mathcal{B}(p ; q)$ could serve as examples of algebras that are differentially but not necessarily homologically smooth. A relationship between these two forms of (noncommutative) smoothness is not yet understood.

The construction of a real Dirac operator on $\mathcal{B}(p ; q)$ presented above is purely algebraic. An obvious next step is to study analytic aspects of this construction in order to form real spectral triples on $\mathcal{B}(p ; q)$ (as opposed to algebraic Dirac operators with a real structure hitherto described). In the first instance one should study the theory of $*$-representations of $\mathcal{B}(p ; q)$, the rudiments of which are outlined in [5] in order to construct a suitable Hilbert space. This process might force one to impose additional conditions on the polynomial $p$. One can also follow the procedure of Beggs and Majid employed successfully in [2] in the case of the quantum sphere and the quantum disc. This procedure makes use of the integral on the studied algebra. The integral $\Lambda$ on $\mathcal{A}(p ; q)$ constructed in Proposition 4.7 can be restricted to $\mathcal{B}(p ; q)$. Since the integral space is finite but not one-dimensional, before the restriction of $\Lambda$ can be used to obtain an inner product, a suitable hermitian inner product on the integral space need be constructed.

These are topics for future work.

\section{Acknowledgements}

The author would like to express his gratitude to the referees for many helpful and detailed comments and suggestions.

\section{References}

[1] Bavula V., Tensor homological minimal algebras, global dimension of the tensor product of algebras and of generalized Weyl algebras, Bull. Sci. Math. 120 (1996), 293-335.

[2] Beggs E.J., Majid S., Spectral triples from bimodule connections and Chern connections, arXiv:1508.04808.

[3] Beggs E.J., Smith S.P., Non-commutative complex differential geometry, J. Geom. Phys. 72 (2013), 7-33, arXiv:1209.3595.

[4] Brzeziński T., Non-commutative connections of the second kind, J. Algebra Appl. 7 (2008), 557-573, arXiv:0802.0445. 
[5] Brzeziński T., Circle and line bundles over generalized Weyl algebras, Algebr. Represent. Theory 19 (2016), 57-69, arXiv:1405.3105.

[6] Brzeziński T., El Kaoutit L., Lomp C., Non-commutative integral forms and twisted multi-derivations, J. Noncommut. Geom. 4 (2010), 289-312, arXiv:0901.2710.

[7] Brzeziński T., Fairfax S.A., Quantum teardrops, Comm. Math. Phys. 316 (2012), 151-170, arXiv:1107.1417.

[8] Brzeziński T., Hajac P.M., The Chern-Galois character, C. R. Math. Acad. Sci. Paris 338 (2004), 113-116, math.KT/0306436.

[9] Brzeziński T., Majid S., Quantum group gauge theory on quantum spaces, Comm. Math. Phys. 157 (1993), 591-638, Erratum, Comm. Math. Phys. 167 (1995), 235-235, hep-th/9208007.

[10] Brzeziński T., Sitarz A., Smooth geometry of the noncommutative pillow, cones and lens spaces, J. Noncommut. Geom., to appear, arXiv:1410.6587.

[11] Connes A., Noncommutative geometry and reality, J. Math. Phys. 36 (1995), 6194-6231.

[12] Dade E.C., Compounding Clifford's theory, Ann. of Math. 91 (1970), 236-290.

[13] Dade E.C., Group-graded rings and modules, Math. Z. 174 (1980), 241-262.

[14] Hajac P.M., Strong connections on quantum principal bundles, Comm. Math. Phys. 182 (1996), 579-617, hep-th/9406129.

[15] Hong J.H., Szymański W., Quantum lens spaces and graph algebras, Pacific J. Math. 211 (2003), 249-263.

[16] Khalkhali M., Landi G., van Suijlekom W.D., Holomorphic structures on the quantum projective line, Int. Math. Res. Not. 2011 (2011), 851-884, arXiv:0907.0154.

[17] Krähmer U., On the Hochschild (co)homology of quantum homogeneous spaces, Israel J. Math. 189 (2012), $237-266$.

[18] Liu L., Homological smoothness and deformations of generalized Weyl algebras, Israel J. Math. 209 (2015), 949-992, arXiv:1304.7117.

[19] Lunts V.A., Rosenberg A.L., Kashiwara theorem for hyperbolic algebras, Preprint MPIM-1999-82, 1999.

[20] Majid S., Noncommutative Riemannian and spin geometry of the standard $q$-sphere, Comm. Math. Phys. 256 (2005), 255-285, math.QA/0307351.

[21] Năstăsescu C., van Oystaeyen F., Graded ring theory, North-Holland Mathematical Library, Vol. 28, NorthHolland Publishing Co., Amsterdam - New York, 1982.

[22] Podleś P., Quantum spheres, Lett. Math. Phys. 14 (1987), 193-202.

[23] van den Bergh M., A relation between Hochschild homology and cohomology for Gorenstein rings, Proc. Amer. Math. Soc. 126 (1998), 1345-1348.

[24] Woronowicz S.L., Compact matrix pseudogroups, Comm. Math. Phys. 111 (1987), 613-665.

[25] Woronowicz S.L., Twisted SU(2) group. An example of a noncommutative differential calculus, Publ. Res. Inst. Math. Sci. 23 (1987), 117-181. 